\title{
EPR INVESTIGATIONS OF MODEL NEUROMELANINS
}

\author{
D. SKRZYPEK ${ }^{a, *}$, A. DZIERżĘGA-LęCZnAR ${ }^{b}$ AND J. ZIóŁKoWSKA ${ }^{a}$ \\ ${ }^{a}$ Institute of Physics, University of Silesia, Uniwersytecka 4, 40-007 Katowice, Poland \\ ${ }^{b}$ Faculty of Pharmacy, Silesian Medical Academy \\ Narcyzów 1, 41-200 Sosnowiec, Poland
}

The aim of this work was to compare free radical properties of some model neuromelanins obtained from dopamine and its mixture with 5-S-cysteinyldopamine in various molar ratios. The EPR method was used in these investigations; the samples were detected at $\mathrm{X}$ and $\mathrm{K}$ bands. The parameters of EPR spectra, free radicals concentrations in air and in vacuum, the influence of microwave power and temperature on intensity of resonance signals were measured.

PACS numbers: $76.30 . \mathrm{Rn}, 87.15 .-\mathrm{v}$

\section{Introduction}

Most dopaminergic neurons of human brain substantia nigra are pigmented with neuromelanin. It seems that this biopolimer may be actively involved in neurodegenerative processes that occur during natural ageing and Parkinson's disease [1]. Numerous data indicate that the nigral pigment consists of indole-type (eumelanin) and benzothiazine-type (pheomelanin) subunits, derived oxidatively from dopamine and 5-S-cysteinyldopamine, respectively $[2,3]$. EPR studies evidenced that both structural constituents of natural neuromelanin are the sources of distinct kind of stable organic radicals [4].

It has been found previously that pure eumelanin synthesized from dopamine is characterized by o-semiquinone oxygen radicals, whereas 5-S-cysteinyldopamine-derived pheomelanin contains nitrogen-centered semiquinoneimine radicals [5]. The aim of this work was to compare free radical properties of some model neuromelanins obtained from dopamine and its mixture with 5-S-cysteinyldopamine in various molar ratios.

\footnotetext{
*corresponding author; e-mail: dskrzyp@us.edu.pl
} 


\section{Materials and methods}

5-S-cysteinyldopamine was obtained by tyrosinase-catalyzed oxidation of dopamine in the presence of cysteine and purified by ion-exchange chromatography [6].

Model neuromelanins were synthesized by autooxidative polymerization of dopamine (DA) and 5-S-cysteinyldopamine (5-S-CDA), mixed in various molar ratios, in $50 \mathrm{mM}$ sodium phosphate buffer $(\mathrm{pH} 7.0)$ at $310 \mathrm{~K}$ with vigorous stirring and light protection. Pure DA-melanin and 5-S-cysDA-melanin were also synthesized using the same procedure.

Spectra EPR at X band $(\sim 9 \mathrm{GHz})$ were recorded with a standard Radiopan spectrometer system. At $\mathrm{K}$ band $(\sim 22 \mathrm{GHz})$ a $\mathrm{MB} 257$ microwave bridge was used with a spectrometer.

\section{Results and discussion}

Free radical properties of synthetic pheomelanins obtained from various cysteinyl adducts of dopamine, as well as those of eu-/pheo-copolymer from dopamine and 5- S-cysteinyldopamine were compared previously at $\mathrm{X}$ band [5]. For all the studied samples, complex EPR spectra with a poorly resolved hyperfine structure were recorded. According to Sealy et al. [7], the EPR signals were characterized additionally by " $R$ "-factor, defined as the height ratio of central high-field to low-field maximum. Such a parameter seems to be helpful for determination of the relative content of eu- and pheo-type components in the melanin pigments of mixed character.

The presence of nitrogen centered free radicals with $g$-values of 2.0052 was proved for pure pheomelanin from 5-S-cysteinyldopamine. For eu-/pheo-copolymer, derived oxidatively from an equimolar mixture of dopamine and 5-S-cysteinyldopamine, lowering $g$-value to 2.0046 , as well as a significant increase in $R$-factor could be observed. Hence, it was concluded that oxygen free radicals of eumelanin component were predominant in this kind of biopolymer.

It was evidenced by degradative studies [3] that human substantia nigra neuromelanin could contain variable amounts of pheomelanin component. The natural pigment should be modeled then by synthetic copolymers derived from dopamine and 5-S-cysteinyldopamine rather than by pure dopamine melanin.

It can be assumed that, depending on its chemical composition, human neuromelanin may be involved in some patho-physiological processes with various efficiency. The ratio of pheomelanin to eumelanin subunits implies not only intrinsic structure and aggregation mode of the biopolymer, but also its free radical type and concentration. The EPR spectra of free radicals in synthetic dopamine-melanin and 5-S-cysteinyldopamine-melanin are distinguishable [5]. The DA-mel has a single line, whereas the 5-S-CDA-mel, in which the adduct is sulfur, has a spectrum characteristic of an immobilized radical that is partly nitrogen centered, most probably a semiquinonimine. In all copolymers (Fig. 1) EPR signals similar to those found for 5-S-CDA melanin are present.

The parameters of the EPR spectra for the investigated model neuromelanins are given in the Table. The samples of pheomelanin-type have $g_{\text {app }}$ values 


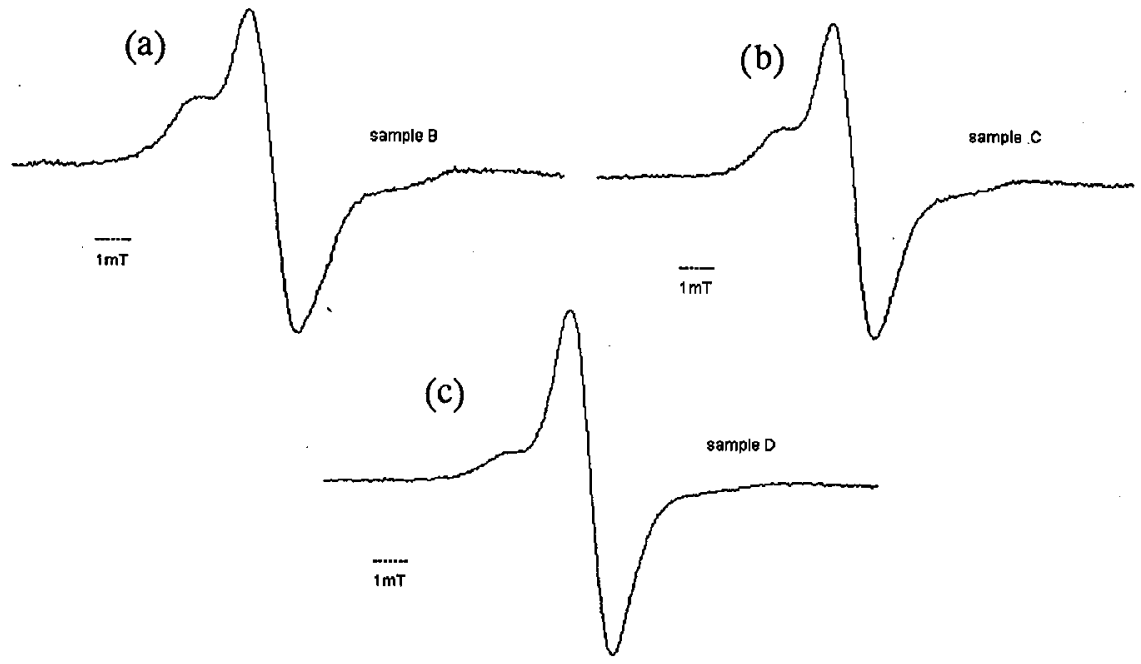

Fig. 1. EPR spectra of DA/5-S-CDA-mel: molar ratio - (2.5:7.5) (a), (1:1) (b), $(7.5: 2.5)(\mathrm{c})$.

TABLE

The parameters of the EPR spectra: $R$-factor, $g_{\text {app }}$ (the apparent $g$-factor obtained from X-band data was measured at the crossover points of the first-derivative spectrum), $g_{z}$ and $A_{z}$ ( $g$ and $A$ are tensors - see Eq. (1)), for the studied synthetic neuromelanins.

\begin{tabular}{c|l|c|c|c|c}
\hline \hline Sample & \multicolumn{1}{|c|}{ Melanin from } & $\begin{array}{c}R- \\
\text { factor }\end{array}$ & $\begin{array}{c}g_{\mathrm{app}} \\
\pm 0.0005\end{array}$ & $\begin{array}{c}g_{z} \\
\pm 0.0005\end{array}$ & $\begin{array}{c}A_{z}[\mathrm{mT}] \\
\pm 0.01\end{array}$ \\
\hline $\mathrm{A}$ & $\begin{array}{l}\text { 5-S-cysteinyldopamine-melanin } \\
\text { 5-S-CDA-mel }\end{array}$ & 2 & 2.0050 & 2.0032 & 1.0 \\
\hline $\mathrm{B}$ & $\begin{array}{l}\text { Dopamine-melanin and } \\
\text { 5-S-cysteinyldopamine-melanin } \\
\text { (molar ratio 2.5 : 7.5) } \\
\text { DA/5-S-CDA-mel (2.5 : 7.5) }\end{array}$ & 2.2 & 2.0048 & 2.0031 & 1.07 \\
$\mathrm{C}$ & $\begin{array}{l}\text { Dopamine melanin and } \\
\text { 5-S-cysteinyldopamine-melanin } \\
\text { (molar ratio 1:1) }\end{array}$ & 3.2 & 2.0043 & 2.0030 & 1.1 \\
\hline DA/5-S-CDA-mel (1:1) & $\begin{array}{l}\text { Dopamine melanin and } \\
\text { 5-S-cysteinyldopamine-melanin } \\
\text { (molar ratio 7.5 : 2.5) } \\
\text { DA/5-S-CDA-mel (7.5:2.5) }\end{array}$ & 5.7 & 2.0043 & 2.0031 & 1.01 \\
\hline $\mathrm{E}$ & Dopamine-melanin DA-mel & - & 2.0038 & - & -
\end{tabular}


characteristic of free radicals nitrogen-centered. The mixed type melanin exhibit relatively higher $R$-factor compared to pure pheomelanin (sample $\mathrm{A}$ ).

\subsection{EPR measurement at $X(9 \mathrm{GHz})$ and $K($ $22 \mathrm{GHz})$ bands}

We also recorded EPR spectra of synthetic neuromelanins at $\mathrm{K}$ band. Figure 2 shows the spectra obtained at various microwave frequencies for 5-S-CDA-mel (for example).
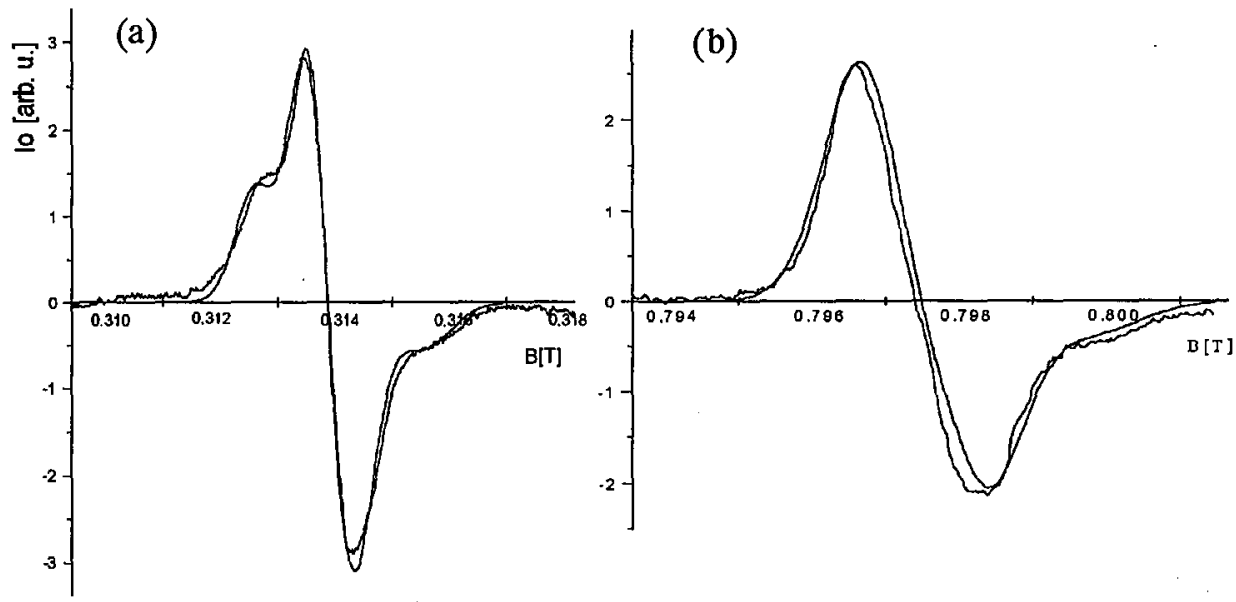

Fig. 2. EPR spectra of model 5-S-CDA-mel at X (a) and K (b) bands. The smooth lines mean the fitting to $\mathrm{Eq}$. (2).

${ }^{14} \mathrm{~N}$ hyperfine coupling in $\pi$ radicals is generally axial and anisotropic $\left(A_{z}>A_{\perp}\right), g$-factor is also axial. The Zeeman splitting is proportional to the microwave frequency because the EPR resonance condition derived from the first-order perturbation theory for the hyperfine term while neglecting the fine structure, quadrupolar and nuclear Zeeman terms is as follows:

$$
h \nu=g \mu_{\mathrm{B}} B+A m
$$

where

$$
\begin{aligned}
& g=\left(g_{1}^{2} \sin ^{2} \theta \sin ^{2} \varphi+g_{2}^{2} \sin ^{2} \theta \cos ^{2} \varphi+g_{3}^{2} \cos ^{2} \theta\right)^{1 / 2}, \\
& A=\left(A_{1}^{2} g_{1}^{2} \sin ^{2} \theta \sin ^{2} \varphi+A_{2}^{2} g_{2}^{2} \sin ^{2} \theta \cos ^{2} \varphi+A_{3}^{2} g_{3}^{2} \cos ^{2} \varphi\right)^{1 / 2} .
\end{aligned}
$$

The critical points of the powder pattern arising from Eq. (1) have been calculated for the case of axial symmetry. When the magnetic field is swept and the frequency held constant, the critical points occur at the following fields:

$$
B_{i}=\left(h \nu_{0} / g_{i} \mu_{\mathrm{B}}\right)-\left(A_{i} m / g_{i} \mu_{\mathrm{B}}\right)
$$

The comparison of EPR measurements at $\mathrm{X}$ and $\mathrm{K}$ bands shows that the complex spectra for 5-S-CDA-mel and copolymers DA/5-S-CDA-mel are the case of axial symmetry. The estimation is based on the comparison of experimental spectra with the spectra calculated from Eq. (2). The results obtained by this procedure are shown in the Table. We concluded that the same kind of free radicals in all investigated model pheomelanins is present, as those found for 5-S-CDA-melanin. 


\subsection{Microwave power saturation}

EPR studies were performed for model neuromelanins in the presence or not of atmospheric oxygen in the environment of samples. Progressive microwave power saturation of EPR signal was used to obtain information about the participation of oxygen molecules in the changes occurring in the free radicals population and spin relaxation processes.

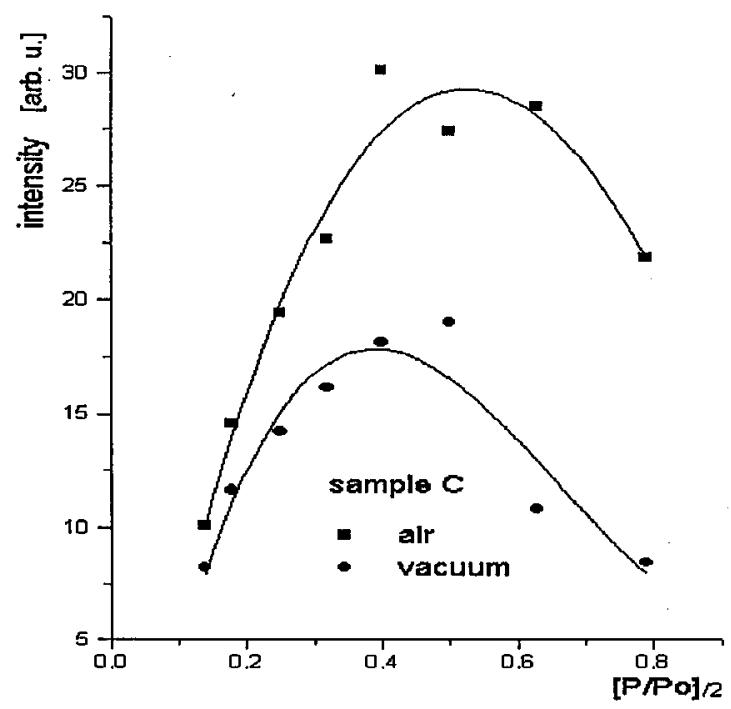

Fig. 3. The influence of microwave power on the intensities of the EPR signals of DA/5-S-CDA-mel: sample C. $P_{0}$ and $P$ - total microwave power produced by klystron and used microwave power, respectively. The intensities were normalized.

Figure 3 shows that the intensity of EPR signal $(I)$ saturates with the microwave power $\left(P^{1 / 2}\right)$ leading to deviations of linearity between $I$ and $P^{1 / 2}$. Similar figures were obtained for all model neuromelanins. The higher values of $P^{1 / 2}$ (that are inversaly proportional to $T_{1} T_{2}-$ the product of transversal and longitudinal relaxation times) are obtained when oxygen is present. The concentration of paramagnetic centers in DA-mel is not dependent on the presence of molecular oxygen, whereas air removing from other model neuromelanins leads to the decrease in paramagnetic centers concentrations. However, it seems that in these cases the accessibility of oxygen to paramagnetic centers is low.

\subsection{Temperature investigations of model neuromelanins}

The experiments were performed at a series of temperatures from $300 \mathrm{~K}$ to $150 \mathrm{~K}$. Figure 4 shows the product $I T$ as a function of temperature $T$ ( $I$ was obtained by a double integration of the first derivative spectra - it was necessary to relate signal amplitudes to paramagnetic susceptibility). The data were normalized to $T=T_{R}$. The susceptibility of normal paramagnetics is universally proportional 

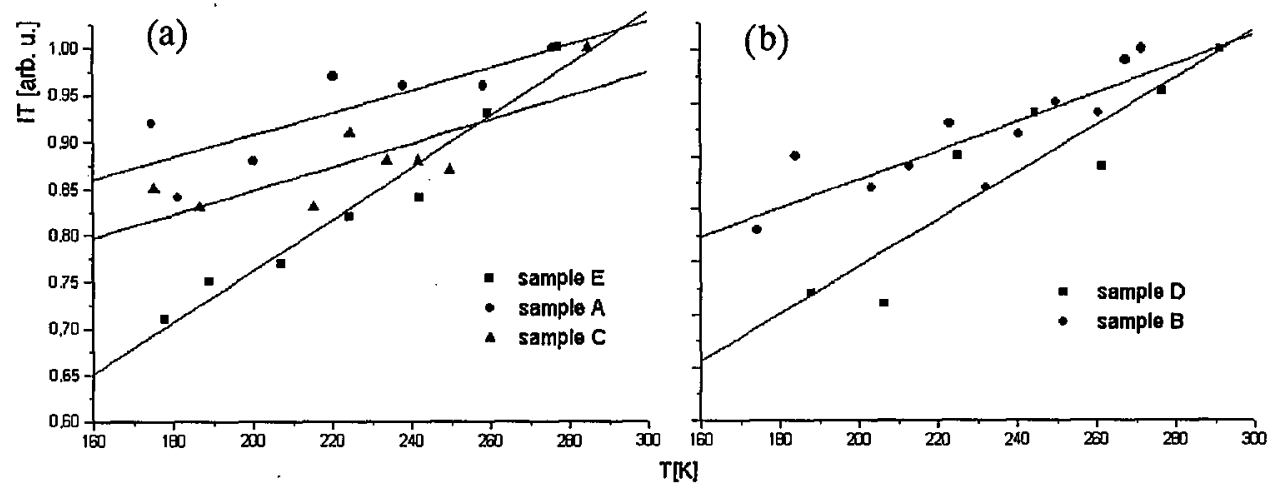

Fig. 4. Temperature dependences $I T$ vs. $T$ for model neuromelanin: (a) samples $E, A, C$; (b) samples D,B.

to the temperature $T$. Thus the product $I T$ is constant vs. $T$. We believe that the distinct changes might be interpreted in terms of thermal induced radicals.

Our experiments (data not shown) also indicate that temperature dependences of $I T$ were not affected by the presence of atmospheric oxygen in the sample environment.

\section{Conclusions}

It was evidenced [3] that human substantia nigra neuromelanin could contain variable amounts of pheomelanin component. The natural pigment should be modeled then by synthetic copolymers derived from dopamine and 5-S-cysteinyldopamine rather, than by pure dopamine melanin.

We investigated free radical properties of these copolymers. By monitoring the signal intensities and progressive microwave power saturation it was ascertained that model neuromelanins interact with molecular oxygen. The estimation for experimental results at $\mathrm{X}$ and $\mathrm{K}$ bands shows that complex EPR spectra for synthetic neuromelanin are the case of axial symmetry. The measurements performed at a series of temperatures may suggest the presence of thermal induced radicals.

\section{References}

[1] M.B.H. Youdim, D. Ben-Shachar, P. Riederer, J. Neur. Transm. 43, 113 (1994).

[2] R. Carstam, C. Brinck, A. Hindemith-Augustsson, H. Rorsman, E. Rosengren, Biochim. Biophys. Acta 1097, 152 (1991).

[3] G. Odh, R. Carstam, J. Paulson, A. Wittbjer, E. Rosengren, H. Rorsman, J. Neurochem. 62, 2030 (1994).

[4] W.S. Enochs, M.J. Nilges, H.M. Swartz, J. Neurochem. 61, 68 (1993).

[5] A. Dzierżęga-Lęcznar, B. Pilawa, K. Stępień, T. Wilczok, Nucleonika 42, 343 (1997).

[6] S. Ito, K. Fujita, M. Yoshioka, D. Sienko, T. Nagatsu, J. Chromatograph. 375, 134 (1986).

[7] R.C. Sealy, J.S. Hyde, C.C. Felix, I.A. Menon, G. Prota, Science 217, 545 (1982). 